\title{
BIOCHEMICAL AND HORMONAL PARAMETERS IN PATIENTS PRESENTING WITH HIRSUTISM
}

Yıldız Okuturlar*, Samet Sayılan*, Meral Mert"* , Ozlem Soyluk**, Ezgi Erdogan*, Ozlem Harmankaya*, Abdulbaki Kumbasar ${ }^{*}$

*Bakirkoy Dr. Sadi Konuk Education and Research Hospital,Department of Internal Medicine, Istanbul, Turkey

**Bakirkoy Dr. Sadi Konuk Education and Research Hospital, Department of Endocrinology and Metabolism, Istanbul, Turkey

Introduction:

It was aimed to evaluate the biochemical and hormonal parameters in female patients with and without polycystic ovary and presenting with symptoms of hirsutism.

\section{Methods:}

\section{Results:}

In this study, 217 female patients presenting with symptoms of hirsutism were enrolled.

Seventy two female patients having polycystic ovaries by ultrasonography were named as group-1 and 145 female patients with normal ovaries were named as group-2. Follicle stimulating hormone (FSH), Luteinizing hormone (LH), plasma total and free testosterone, dehydroepiandrosteronesulfate (DHEA-S), 17hydroxyprogesterone (17-OH Prog), glucose, homeostasis model assessment (HOMA-IR), haemoglobin A1c (HbA1c), total cholesterol, triglyceride, low- density lipoprotein cholesterol (LDL-C), high-density lipoprotein cholesterol (HDL-C) were recorded.

While there was statistically significant difference between total testosterone $(0.62$ $\pm 0.260 .56 \pm 0.24)$ and LH/FSH ratio $(1.54 \pm 1.21,1.18 \pm 0.92)$ in Groups $1 \& 2$ (p $=0.09$ in both), there were no differences among the values of free testosterone, $\mathrm{LH}, \mathrm{FSH}, 17-\mathrm{OH}$ Prog and DHEA-S. There were no significant differences in lipid profile, glucose, insulin, HOMA-IR, HbA1c and plasma uric acid levels. While there were positive correlations between $\mathrm{A} 1 \mathrm{C}$ and total testosterone, free testosterone and HOMA-IR, HbA1C and insulin in Group-1, there were negative correlations between LDL-C and total testosterone, HDL-C and free testosterone in Group-2. There was a positive correlation between HOMA-IR and insulin in LH/FSH ratio only in Group-2.

Hirsutism cases should be closely monitored in terms of metabolic parameters and cardiovascular risk factors. While evaluating hormone profile in these patients, it may be appropriate to conduct research in terms of glucose metabolism disorders and dyslipidaemia. 